\title{
Microwave Kinetic Inductance Detector (MKID) Camera Testing for Submillimeter Astronomy
}

\author{
N.G. Czakon*, A. Vayonakis*, J. Schlaerth ${ }^{\dagger}$, M.I. Hollister*, S. Golwala*, P.K. \\ Day $^{* *}$, J.-S. Gao*, J. Glenn ${ }^{\dagger}$, H. LeDuc**, P.R. Maloney ${ }^{\dagger}$, B. Mazin ${ }^{\dagger}$, O. Noroozian*, \\ H. T. Nguyen**, J. Sayers*, J. E. Vaillancourt* and J. Zmuidzinas* \\ ${ }^{*}$ California Institute of Technology, Pasadena, CA 91125 \\ 'University of Colorado, Boulder, CO 80309 \\ ** Jet Propulsion Laboratory, Pasadena, CA 91109 \\ $¥$ University of California, Santa Barbara, CA 93106
}

\begin{abstract}
Developing kilopixel focal planes for incoherent submm- and mm-wave detectors remains challenging due to either the large hardware overhead or the complexity of multiplexing standard detectors. Microwave kinetic inductance detectors (MKIDs) provide a efficient means to produce fully lithographic background-limited kilopixel focal planes. We are constructing an MKID-based camera for the Caltech Submillimeter Observatory with 576 spatial pixels each simultaneously sensitive in 4 bands at 230,300,350, and $400 \mathrm{GHz}$. The novelty of MKIDs has required us to develop new techniques for detector characterization. We have measured quasiparticle lifetimes and resonator Qs for detector bath temperatures between $200 \mathrm{mK}$ and $400 \mathrm{mK}$. Equivalent lifetime measurements were made by coupling energy into the resonators either optically or by driving the third harmonic of the resonator. To determine optical loading, we use both lifetime and internal Q measurements, which range between 15,000 and 30,000 for our resonators. Spectral bandpass measurements confirm the placement of the 230 and $350 \mathrm{GHz}$ bands. Additionally, beam maps measurements conform to expectations. The same device design has been characterized on both sapphire and silicon substrates, and for different detector geometries. We also report on the incorporation of new shielding to reduce detector sensitivity to local magnetic fields.
\end{abstract}

Keywords: submillimeter instrumentation, kinetic inductance detectors PACS: $95.55 . \mathrm{Br}$

\section{INTRODUCTION}

Kinetic inductance detection is a promising technology for sub/millimeter, optical, and x-ray astronomy[1]. Our collaboration is constructing one of the first astronomical instruments using these detectors, MKIDCam, which is to be commissioned in 2010 at the Caltech Submillimeter Observatory (CSO) on Mauna Kea in Hawaii. The final camera will have 576 spatial pixels-each pixel sensitive to 4 discrete bands for a total of 2304 resonators.

In preparation for the final focal plane design, we have constructed a smaller demonstration camera, DemoCam, and conducted testing on a simplified 16-pixel, 2-color device. Many aspects of the camera's detectors are highly sensitive to fabrication processes and resonator fine tuning can only be accomplished with empirical measurements. Our optical detector characterization is extensive: beam maps to determine beam shapes as defined by the device's optical chain, hot-cold load measurements to determine responsivity, and fourier transform spectrography to characterize the lithographic bandpass filters and corresponding millimeter wave feed network. Additionally, quasiparticle lifetimes and resonator properties are measured for a range of detector temperatures and readout powers. Based on these results we have modified the
DemoCam design to include better magnetic shielding, increased resonator uniformity, and lowered noise. The modified device has three different colors as well as dark resonators for each of the 36 pixels. It also contains several film resistivity test structures and several high kinetic inductance fraction test resonators to independently measure film resistivity and kinetic inductance, necessary for interpreting device test results. The modified DemoCam instrument will be taken to the CSO for a telescope engineering run in Fall of 2009.

\section{PRINCIPLE OF OPERATION}

To fully appreciate microwave kinetic inductance detectors (MKIDs) requires a review of superconductivity and microwave engineering. Specific details of our instrument and associated detectors can be found in other papers presented in this conference $[2,3,4,5]$.

Close to resonance, MKIDs behave as lumpedelement LC resonators. Take the series lumped-element resonance circuit in figure 1 which has an effective impedance of $Z=1 / j \omega C+j \omega L+R$. The resonance frequency is $\omega_{0}=1 / \sqrt{L C}$ and the quality factor, $Q=1 / \omega_{0} R C$, characterizes the line width of the res- 


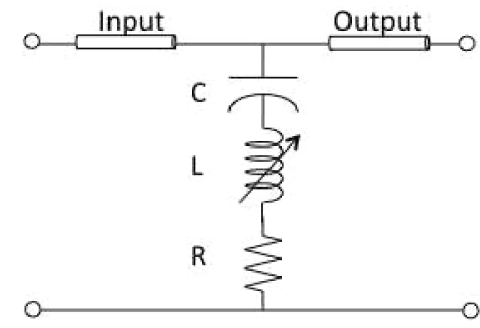

FIGURE 1. MKID as a lumped-element resonant circuit (make the inductance variable)

onator. The total inductance of an MKID is determined both by its shape (geometric inductance) and by its quasiparticle density (kinetic inductance).

MKIDs must be able to effectively couple collected signal into breaking a resonator's Cooper pairs into quasiparticles. With the MKIDCam light is received by an individual pixel's 32-element phased-antenna array and is coherently combined by a binary tree network. Four parallel on-chip bandpass filters define the spectral bands, each with its own MKID. Transmitted energy is trapped in an aluminum section of each resonator which has a lower gap frequency $(\sim 90 \mathrm{GHz})$ than the signal itself ( $\sim 200-400 \mathrm{GHz})$, creating quasiparticles and changing the kinetic inductance of the resonator. The associated shift in $\omega_{0}$ and $Q$ is recorded by our readout system.

\section{THE MKID TESTBED}

Our Al-Nb hybrid resonator MKIDs are typically operated at a base temperature of $230 \mathrm{mK}$, sufficiently below the critical temperature of aluminum, $1.2 \mathrm{~K}$, that quasiparticles created by the quiescent optical load dominate the overall quasiparticle population. We characterize the resonator properties by doing dark measurements, with the device blanked off at the sub-Kelvin stage, varying the temperature loads between 230 and $400 \mathrm{mK}$ to vary the thermal quasiparticle population.

Our IQ readout schematic is presented in figure 2. The transmitted first harmonic readout signal from the MKID array is amplified once by a low-noise HEMT at $4 \mathrm{~K}$ and again by a room temperature amplifier. This signal is mixed down to DC with part of the reference signal that did not pass through the device. To measure the quasiparticles lifetime in the MKIDs, discussed in detail below, we combine chopped resonator third-harmonic power with the first harmonic bias power. Measurement results are given below.

\section{Q Measurements}

One of the standard techniques that we use to characterize the load on a resonator is to measure its quality factor.* The interested reader is referred to two excellent sources that thoroughly describe the art of measuring $\mathrm{Q}[7,8]$. The quality factor is the ratio of the total power inside of a resonator to the amount of energy lost per cycle. For our purposes, we categorize these losses in two categories: coupling loss and internal loss. Coupling loss, $P_{\text {coupling }}$, is the amount of power lost through the coupling capacitor to the feedline and internal loss, $P_{\text {int }}$ accounts for all other loss. Coupling loss and internal loss can be expressed as two different quality factors, $Q_{c}$ and $Q_{i n t}$ which add as the reciprocal of the sum of their reciprocals, i.e.,

$$
\frac{1}{Q_{\text {total }}}=\frac{P_{\text {int }}+P_{\text {coupling }}}{P_{\text {total }}}=\frac{1}{Q_{c}}+\frac{1}{Q_{\text {int }}}
$$

These Qs can be determined by fitting the IQ loop of our transmitted signal to a circle which is modeled in reference [7] as

$$
t_{21}(f)=a e^{-j \omega \tau}\left[1-\frac{Q_{r} / Q_{c} e^{j \phi_{0}}}{1+2 j Q \frac{\omega-\omega_{0}}{\omega}}\right]
$$

where $t_{21}$ is the complex transmitted signal measured by the network analyzer. Below are recent Q measurements made with various optical loads for DemoCam. The very high dark value of $Q_{i n t}$ indicates that $Q_{i n t}$ under load is dominated by optically generated quasiparticles. As explained in [5], signal-to-noise is optimal near the condition $Q_{i n t} \approx Q_{c}$ which in this case is best for a $77 \mathrm{~K}$ load. This is by design, we find that a $77 \mathrm{~K}$ load is typical of the sky load at the telescope and our match is sufficient.

\section{Lifetime Measurements}

After energy breaks Cooper pairs in a resonator, the characteristic time that it takes for the quasiparticles to recombine is known as the lifetime. There are two principle methods that we use to measure lifetimes: third harmonic excitation via the microwave transmission line and millimeter wave excitation via an external source. We measure lifetimes for various base temperatures and excitiation powers. A schematic of the third harmonic excitation method is in figure 2 and a sample measurement is given in figure 3 . The chopped third harmonic frequency is combined with the first harmonic in the transmission line.

*We also measure frequency shift as a function of temperature, though this is less useful for determining total loading because there is no zero load reference point as is the case with Q-measurements. 


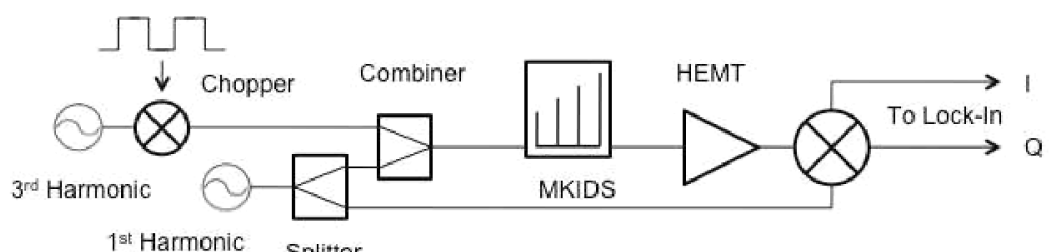

FIGURE 2. Third harmonic excitation scheme for lifetime measurements

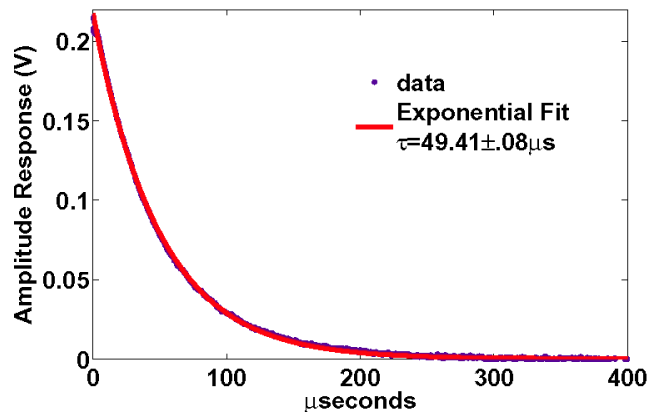

FIGURE 3. A sample fit to data collected by means of third harmonic excitation

When the third harmonic is switched on, it creates a steady-state of quasiparticles in the resonator which will recombine in a characteristic time after the third harmonic is switched off. This can be modeled effectively by a simple exponential fit. Although the method for the creation of quasiparticles is not fully understood, we suspect that it is the result of indirect Cooper pair breaking by energetic quasiparticles that have been heated by the incoming RF power.

\section{Spectral Measurements}

Multi-color operation is achieved with parallel on-chip bandpass filters. These replace standard metal mesh filters routinely used in submillimeter wave detectors. The filters are simple LC filters requiring a minimal number of masks during fabrication. We have verified with Fourier transform spectroscopy, see figure 4, the bandpasses for two of our spectral bands. In the final camera there will be an additional $400 \mathrm{GHz}$ band and the current $250 \mathrm{GHz}$ band will be replaced with 230 $\mathrm{GHz}$ and $300 \mathrm{GHz}$ centered bands. We are currently investigating the cause of the spectral fringing which is a unique pattern for each pixel. If pixel-specific calibration for the bands is needed, we do not anticipate it being a problem to the operation of the final camera.

\section{Beam Maps}

We have performed beam maps by raster scanning a thermal source at a fixed distance from the window of the instrument. A beam map for a sample resonator is presented in figure 5 . These results qualitatively match beam shapes obtained by physical optics calculations of the system. Relative detector positions in the focal plane can be obtained using the centroids of the beam maps and confirmed a low-level of wide-angle pickup.

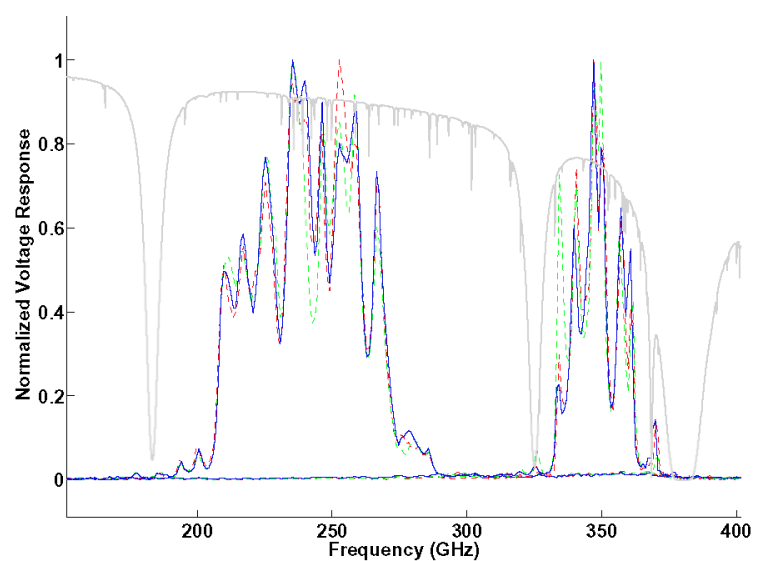

FIGURE 4. Spectral measurements of six different resonators: three resonators in the $250 \mathrm{GHz}$ band and three resonators in the $350 \mathrm{GHz}$ band. A plot of atmospheric transmission at $1.5 \mathrm{~mm}$ precipitable water is shown in grey for comparison.

\section{Magnetic Shielding}

One of the lessons learned from the first engineering run of DemoCam[9] is that good magnetic shielding is essential for MKID operation. We suspect that magnetic field sensitivity is due to flux focusing into the coplanar waveguide (CPW) because the superconducting ground planes expel magnetic field. Recent reports cite a strong sensitivity of superconducting CPWs to magnetic fields applied perpendicular to plane of the waveguide [10]. Although a constant ambient magnetic field is manageable, the MKIDCam will be commissioned on a rotating telescope and it must handle any variation of the Earth's magnetic field as the instrument slews during observations. Initial testing using a rotating bar magnet demonstrated that resonators primarily respond to applied magnetic field in the phase direction. Quasiparticle generation occurs only when the device is exposed to large magnetic fields. We commissioned a Cryoperm10® magnetic shield from Amuneal. At $4 \mathrm{~K}$ the shield attenuates the axial magnetic field by 400 and the transverse field shielding is order of magnitude higher. We tested MKID sensitivity with the shield in place using a Helmholtz coil. Magnetic field response measurements were made via a digital lock-in method using Helmholtz coils to create a magnetic field of up to $360 \mu \mathrm{T}$. The IQ mixer converted the transmitted signal down to baseband resulting in a stable DC value suitable for sensitive low-noise measurements. Hour-long timestreams of this IQ data were taken for a combination of applied DC fields and 50 and $1000 \mathrm{mHz}$ magnetic field modulation. Fourier transforms were performed on the amplitude data in each case. A sample Fourier-transformed data set is presented in figure 6 for a 1 $\mathrm{Hz}$ modulated $300 \mu \mathrm{T}$ magnetic field. We conclude from our measurements that a resonance frequency shift of about $.5 \mathrm{~Hz}$ is to be expected 

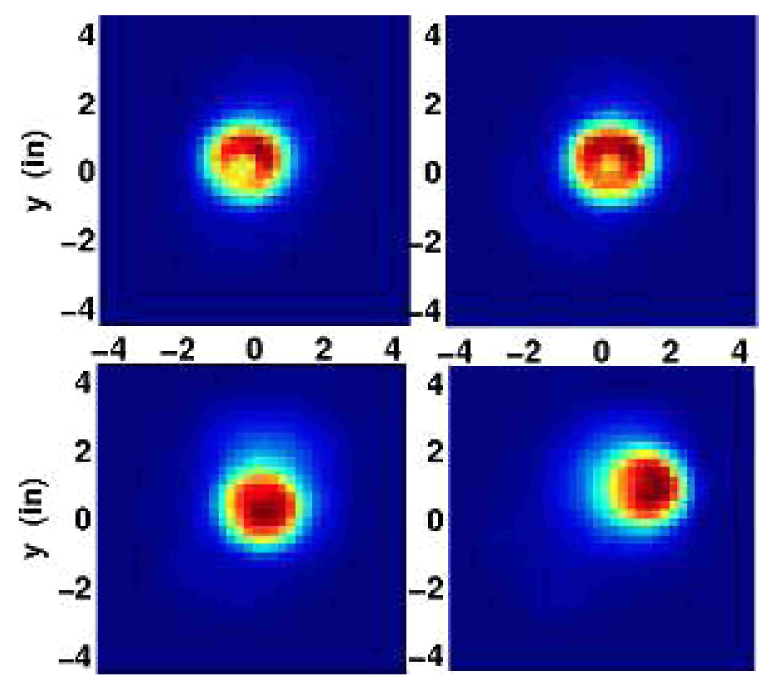

FIGURE 5. Beam maps of four distinct resonators. Note the similarity in the size of the beams. The detailed central structure and shoulders reflect variation in the way the beams propagate through the optics to the beam-mapping source.

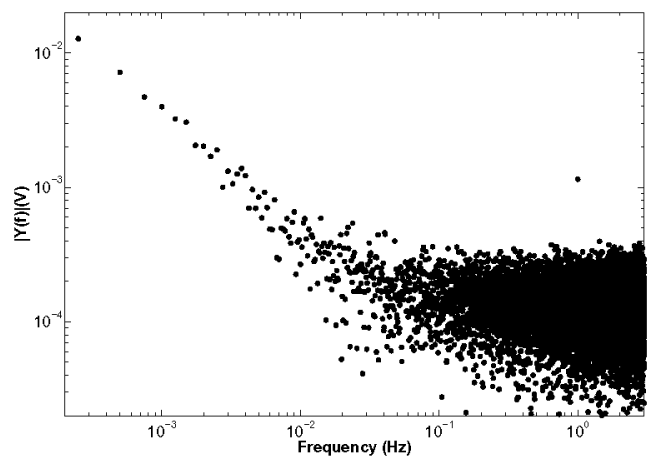

FIGURE 6. Log-log fourier response of a resonator exposed to $300 \mu \mathrm{T}$ AC magnetic field at $1 \mathrm{~Hz}$ averaged over one hour. Even exposed to magnetic fields an order of magnitude larger than the Earth's field averaged for long periods of time, the signal response is quite small.

if our current setup is slewed through the Earth's magnetic field. This is several orders of magnitude better than the previous system and the effect, if any, would only be noticeable on extremely long science integrations.

\section{CONCLUSION}

MKIDCam will be an exciting low-noise high-pixel-count instrument to further sub/millimeter astronomy. Extensive device characterization has been performed on a simplified version of the focal plane. Similar testing is underway on a new focal plane array designed as a prototype for one of the sixteen tiles of the final camera.

\section{REFERENCES}

1. P. Day, H. Leduc, B.Mazin, A. Vayonakis, and J. Zmuidzinas, Nature 425, 817-821 (2003).

2. P. Maloney, this conference (2009).

3. B. Mazin, this conference (2009).

4. O. Noroozian, this conference (2009).

5. J. Schlaerth, this conference (2009).

6. t. t. i. 1. u. f. d. t. 1. b. t. i. n. z. 1. r. p. a. i. t. c. w. Q.-m. We also measure frequency shift as a function of temperature (????).

7. J. Gao, The Physics of Superconducting Microwave Resonators, Ph.D. thesis, California Institute of Technology, Pasadena, CA 91125 (2008).

8. P. Peterson, and S. Anlage, Journal of Applied Physics 84, 3394-3402 (1998)

9. J. Schlaerth, A. Vayonakis, P. Day, J. Glenn, J. Gao, S. Golwala, S. Kumar, H. Leduc, B.Mazin, J. Vaillancourt, and J. Zmuidzinas, Journal of Low Temperature Physics 151, 684-689 (2008).

10. J. Healey, T. Lindstrom, M. S. Colclough, C. M. Muirhead, and A. Y. Tzalenchuk, Applied Physics Letters 93, 043513 (2008) 